\title{
Rapid Assembly of Quinolizidines via Consecutive Nucleophilic Cyclizations onto Activated Amides
}

2011

Vol. 13, No. 16

$4268-4271$

\author{
Guillaume Bélanger, * Gary O’Brien, and Robin Larouche-Gauthier \\ Département de Chimie, Université de Sherbrooke, 2500 Boulevard de l'Université, \\ Sherbrooke, Québec J1K 2R1, Canada
}

guillaume.belanger@usherbrooke.ca

Received June 16, 2011

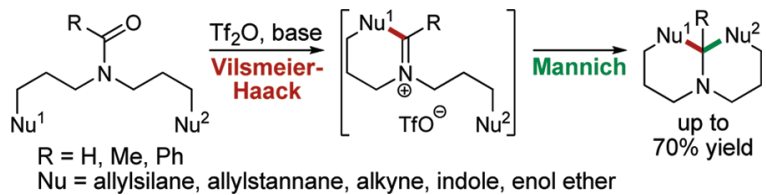

A new approach to the synthesis of quinolizidines involving a cascade of nucleophilic cyclizations triggered by chemoselective amide activation is reported. Particular attention was given to the effect of the nature of the tethered nucleophiles on the cascade of cyclizations. As a result, simple acyclic amides gave rapid access to functionalized quinolizidines bearing either a tertiary or quaternary center at the ring junction. Such a fused bicyclic motif is found in several alkaloids.

Quinolizidines are included within the framework of numerous biologically active and structurally complex alkaloids (Figure 1). Such a ubiquitous framework has attracted the attention of organic synthetic chemists for years in a quest to discover new and efficient ways that would allow for a rapid access to functionalized quinolizidines. Although several monocyclization approaches of appropriately substituted piperidines have been reported, ${ }^{1}$ only a few examples describe the preparation of both cycles in a single operation. The latter strategy offers an important advantage of building molecular complexity in a single sequence from easily accessible acyclic starting materials. One-pot preparation of both cycles of quinolizidines was reported to be the result of either a single reaction, such as the intramolecular iminoacetonitrile $[4+2]$ cycloaddition, $^{2}$ or the result of sequential or tandem processes, such as

(1) For recent reviews on the synthesis of piperidines, see: (a) Buffat, M. Tetrahedron 2004, 60, 1701. (b) Remuson, R.; Gelas-Mialhe, Y. Mini-Rev.Org. Chem. 2008, 5, 193. (c) Whitten, J. P.; Muench, D.; Cube, R. V.; Nyce, P. L.; Baron, B. M.; McDonald, I. A. Bioorg. Med. Chem. Lett. 1991, 1, 441. (d) Harrity, J. P. A.; Provoost, O. Org. Biomol. Chem. 2005, 3, 1349. (e) Hsung, R. P.; Kurdyumov, A. V.; Sydorenko, N. Eur. J. Org. Chem. 2005, 23.

(2) Amos, D. T.; Renslo, A. R.; Danheiser, R. L. J. Am. Chem. Soc. 2003, 125,4970 .

(3) Airiau, E.; Spangenberg, T.; Girard, N.; Breit, B.; Mann, A. Org. Lett. 2010, 12, 528 .

(4) (a) Walther, G. Chem. Ber. 1951, 84, 304. (b) García, D.; Foubelo, F.; Yus, M. Eur. J. Org. Chem. 2010, 2893.

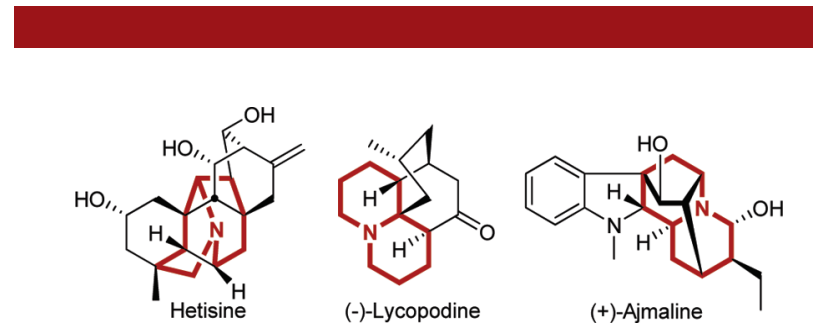

Figure 1. Selected alkaloids bearing a quinolizidine framework (highlighted).

double $\mathrm{N}-\mathrm{C}$ bond formation by reductive aminations, ${ }^{3}$ $\mathrm{N}$-alkylations ${ }^{4}$ or hydroaminations, ${ }^{5}$ double Michael additions, ${ }^{6}$ double ring-closing metathesis reactions, ${ }^{7}$ and a condensation followed by a Mannich (or a PictetSpengler) reaction ${ }^{8}$ (Scheme 1).

(5) Quinet, C.; Sampoux, L.; Markó, I. E. Eur. J. Org. Chem. 2009, 1806

(6) Zou, W.; Sandbhor, M.; Bhasin, M. J. Org. Chem. 2007, 72, 1226.

(7) Ma, S.; Ni, B.; Liang, Z. J. Org. Chem. 2004, 69, 6305.

(8) (a) Auruaym, E.; Spangenberg, T.; Girard, N.; Schoenfelder, A.; Salvadori, J.; Taddei, M.; Mann, A. Chem.-Eur. J. 2008, 14, 10938. (b) Pearson, W. H.; Hembre, E. J. J. Am. Chem. Soc. 2009, 131, 17548. (c) Amorde, S. M.; Jewett, I.; Martin, S. F. Tetrahedron 2009, 65, 3222. (d) Fang, H.; Wu, X.; Nie, L.; Dai, X.; Chen, J.; Cao, W.; Zhao, G. Org. Lett. 2010, 12, 5366 . 
Scheme 1. General Synthetic Approaches to Quinolizidines

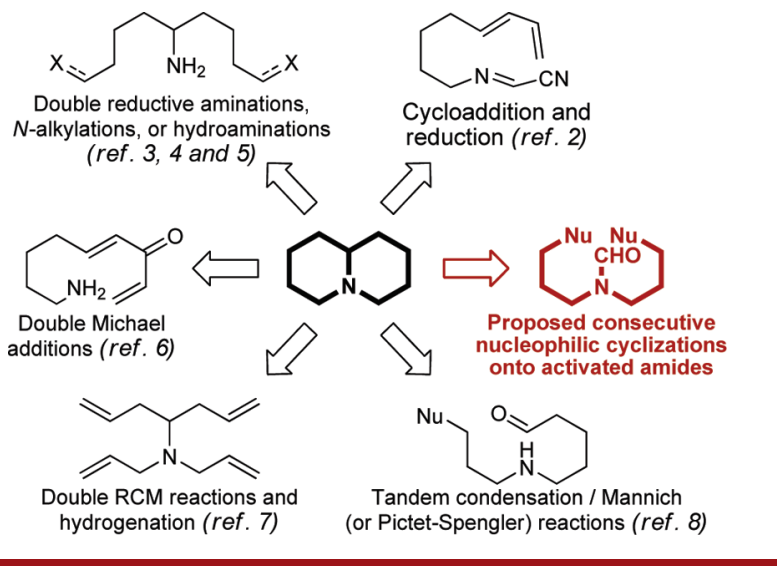

Our group already reported the monocyclization of activated amides that were trapped by various tethered carbon nucleophiles, including enol ethers, enamines, ${ }^{9}$ and allylsilanes. ${ }^{10}$ More recently, we demonstrated that the iminium ions resulting of this first cyclization could be reacted with another tethered nucleophile in a biscyclization sequence. ${ }^{11,12}$ Herein, we describe our expanded synthetic study toward the preparation of quinolizidines. As depicted in Scheme 2, the activation of the amide group in cyclization precursor $\mathbf{1}$ leads to a highly reactive iminium intermediate $\mathbf{2}$ that readily reacts with a nucleophilic tether in a VilsmeierHaack-type cyclization ${ }^{13}$ to generate an iminium ion intermediate $\mathbf{3}$ or $\mathbf{5}$, the identity of which is dependent on the nature of $\mathrm{X}$.

Iminium ion 3 can tautomerize to $\alpha, \beta$-unsaturated iminium ion 4, likely through the corresponding dienamine intermediate. Intermediates $\mathbf{3}-\mathbf{5}$ are then trapped in a Mannich-type cyclization to afford quinolizidines 6-8, respectively. It should be noted that the first iminium ion (2) that bears a carbon of higher oxidation state is more electrophilic than the second iminium ion of lower oxidation state $(\mathbf{3}-\mathbf{5})$ in the reaction cascade. Additionally, in such a system, the more reactive nucleophile always cyclizes first, leaving the less nucleophilic branch to react with the remaining iminium ion of lower electrophilicity. As a consequence, reactivity issues were expected for the second cyclization.

(9) Bélanger, G.; Doré, M.; Ménard, F.; Darsigny, V. J. Org. Chem. 2006, 71,7481

(10) (a) Bélanger, G.; Larouche-Gauthier, R.; Ménard, F.; Nantel, M.; Barabé, F. Org. Lett. 2005, 7, 4431. (b) Bélanger, G.; LaroucheGauthier, R.; Ménard, F.; Nantel, M.; Barabé, F. J. Org. Chem. 2006, 71,704 .

(11) Larouche-Gauthier, R.; Bélanger, G. Org. Lett. 2008, 10, 4501.

(12) The first example of double intramolecular nucleophilic trapping of activated amides was reported by our group (ref 11). The single closest related example is the activation of a carbamate and subsequent nucleophilic trapping. See: Magnus, P.; Gazzard, L.; Hobson, L.; Payne, A. H.; Rainey, T. J.; Westlund, N.; Lynch, V. Tetrahedron 2002, 58, 3423 .

(13) When the nucleophile is an indole or an aryle, the reaction is rather called a Bischler-Napieralski cyclization: Bischler, A.; Napieralski, B. Chem. Ber. 1893, 26, 903.
Scheme 2. Representative Example of Tandem Nucleophilic Cyclizations onto Activated Amides

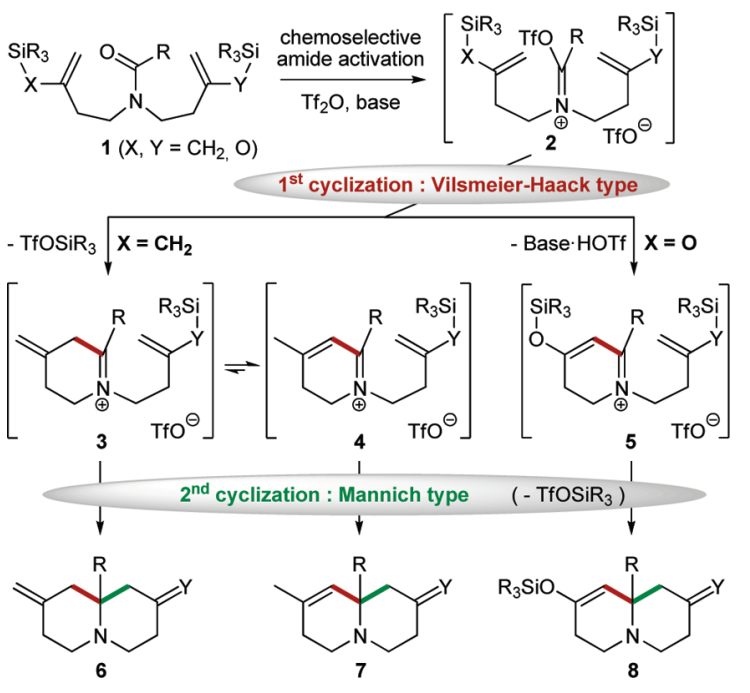

To eliminate the possibility of generating different iminium intermediates, we first tested the tandem nucleophilic cyclizations with a symmetrical substrate (i.e., 1, X, Y $=\mathrm{CH}_{2}$ ) bearing identical nucleophilic branches. When formamide 1a was treated with triflic anhydride $\left(\mathrm{Tf}_{2} \mathrm{O}\right)$ and 2,6-di-tert-butyl-4-methylpyridine (DTBMP) in dichloroethane, quinolizidines $\mathbf{6 a}$ and $7 \mathbf{a}$ were obtained in good yield (entry 1, Table 1). To our delight, this method even allowed for the demanding formation of a quaternary center at the ring junction (entries 2 and 3). As seen in Scheme 2, the first cyclization of activated formamide 1a generated the iminium ion $3\left(\mathrm{R}=\mathrm{H}, \mathrm{Y}=\mathrm{CH}_{2}\right)$ for which the second nucleophilic cyclization was in competition with conjugation to the $\alpha, \beta$-unsaturated iminium ion $4(\mathrm{R}=\mathrm{H}$, $\mathrm{Y}=\mathrm{CH}_{2}$ ), leading to a $1: 1.6$ mixture of quinolizidines $\mathbf{6 a}$ and $7 \mathbf{a}$, respectively. When acetamide $\mathbf{1 b}$ was activated, the more hindered iminium ion $3(\mathrm{R}=\mathrm{Me}, \mathrm{Y}=$ $\mathrm{CH}_{2}$ ) was less reactive and the proportion of conjugated iminium ion $4\left(\mathrm{R}=\mathrm{Me}, \mathrm{Y}=\mathrm{CH}_{2}\right)$ increased, leading to quinolizidine $\mathbf{7 b}$ bearing an endocyclic alkene as the major product.

Another symmetrical substrate $(9)$ was treated in the activation conditions, but unfortunately, no formation of quinolizidine was observed, even after extended reaction time at elevated temperature (entry 4). ${ }^{14}$ Nonetheless, the $\alpha, \beta$-unsaturated iminium ion intermediate $\mathbf{1 0}$ was cleanly formed. ${ }^{15,16}$ The latter arose from a rapid conjugation of

(14) The use of DCM, DCE, and $o$-dichlorobenzene, or the use of cosolvent (benzene, $\mathrm{MeCN}$, or $\mathrm{MeNO}_{2}$ ) to modify the reaction medium polarity affected neither the yields nor the selectivities in any of the entries. In cases where the reaction stopped after the first cyclization (entries 4, 5, and 9), prolonged reaction time and/or higher temperatures resulted in protodesilylation of the remaining nucleophilic branch, along with degradation.

(15) Observed by ${ }^{1} \mathrm{H}$ NMR analysis of an aliquot taken from the reaction mixture. See the Supporting Information for more details. 
Table 1. Quinolizidines via Tandem Nucleophilic Cyclizations onto Activated Amides

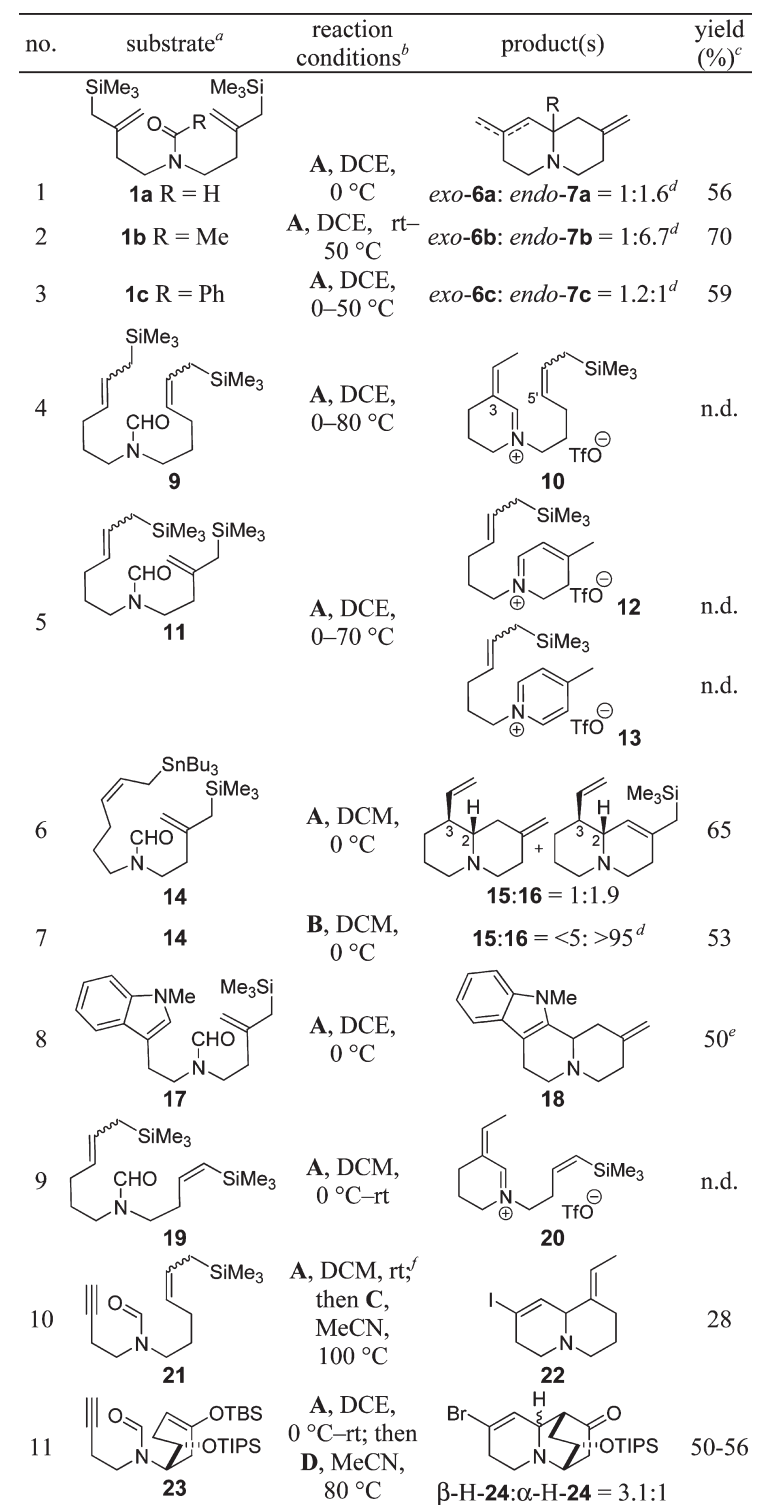

\footnotetext{
${ }^{a}$ Highly convergent syntheses of all substrates are described in the Supporting Information. ${ }^{b}$ Conditions A: $\mathrm{Tf}_{2} \mathrm{O}$ (1.1 equiv), DTBMP (3.0 equiv). Conditions B: $\mathrm{Tf}_{2} \mathrm{O}$ (1.1 equiv), DIPEA ( 5 equiv). Conditions $\mathrm{C}$ : $(n \text {-Bu })_{4} \mathrm{NI}$ (5 equiv). Conditions $\mathrm{D}:(n-\mathrm{Bu})_{4} \mathrm{PBr}\left(2.0\right.$ equiv). ${ }^{c}$ Isolated yields. ${ }^{d}$ Ratio determined by relative integration of characteristic ${ }^{1} \mathrm{H}$ NMR signals of the crude mixture. ${ }^{e} 63 \%$ based on recovered starting material. ${ }^{f}$ Only 1.0 equiv of DTBMP was used.
}

the $\beta, \gamma$-unsaturated iminium ion generated after the first cyclization (cf. $\mathbf{3} \rightarrow \mathbf{4}$, Scheme 2 ) under these conditions.

The failure to generate the desired quinolizidine from substrate 9 contrasts with the smooth sequential cyclizations of substrates $\mathbf{1 a}-\mathbf{c}$ in entries $1-3$. Two factors could have contributed to impede the second cyclization from 9: (1) the steric hindrance generated by substitution

(16) Although the stereochemistry of the trisubstituted double bond of $\mathbf{1 0}$ was not determined for this specific substrate, it was assumed to be cis, as determined for the analogous iminium $\mathbf{2 0}$. at $\mathrm{C}_{3} \alpha$ to iminium carbon in $\mathbf{1 0}$ and/or (2) the steric hindrance generated by substitution on the remaining nucleophilic carbon $\left(\mathrm{C}_{5^{\prime}}\right)$. The answer to this came from treatment of substrate $\mathbf{1 1}$ containing mixed nucleophiles (entry 5): upon activation, we observed ${ }^{15}$ the formation of iminium ion intermediate $\mathbf{1 2}$ that readily aromatized at $70{ }^{\circ} \mathrm{C}$ to yield the pyridinium ion $13^{17}$ with no trace of quinolizidine. Because the iminium ion of $\mathbf{1 2}$ is identical to the iminium ion intermediate obtained from the activation of 1a, we can conclude that the steric hindrance generated by substitution on the remaining nucleophilic carbon has the largest effect on the second cyclization. ${ }^{18}$

Although nonsubstituted allylsilanes are more reactive than their substituted analogues, we could reverse the order of addition by switching to an allylstannane. ${ }^{19}$ Indeed, this subtle change between silane $\mathbf{1 1}$ and stannane $\mathbf{1 4}$ was sufficient to satisfyingly produce quinolizidines $\mathbf{1 5}$ and 16 , in $65 \%$ yield at $0{ }^{\circ} \mathrm{C}$ (entry 6 ).

For the first time throughout this study, we observed the presence of a TMS group in the fully cyclized product (cf. 16), ${ }^{20}$ suggesting that the more nucleophilic allylstannane ${ }^{19}$ branch of $\mathbf{1 4}$ cyclized first. ${ }^{21}$ Trapping of the resulting iminium ion $\mathbf{2 5}$ generated a cationic intermediate $\mathbf{2 6}$ that could be either desilylated to $\mathbf{1 5}$ or deprotonated to 16 (Scheme 3). The use of a weak base (DTBMP) led to a 1.9:1 ratio of $\mathbf{1 5 : 1 6}$ (entry 6, Table 1), whereas the use of a more basic reagent (DIPEA) resulted in the formation of quinolizidine $\mathbf{1 6}$ with an endocyclic alkene as the only detectable product (entry 7 ). ${ }^{22}$ The relative stereochemistry $\left(\mathrm{C}_{2}, \mathrm{C}_{3}\right)$ in $\mathbf{1 5}$ and $\mathbf{1 6}$ is defined by addition of the allylsilane

(17) Since thorough degassing of the reaction mixture to remove oxygen prior to addition of triflic anhydride did not prevent the formation of pyridinium $\mathbf{1 3}$ the oxidation is believed to occur via deprotonation of eniminium 12 and oxidation of the resulting 1,2dihydropyridine by hydride transfer to reduce iminium species in solution and to generate pyridinium 13. For an example of oxidation of 1,2dihydropyridine to pyridinium ion via hydride transfer, see: Cattoe, X.; Bourissoua, D.; Bertrand, G. Tetrahedron 2006, 47, 531.

(18) Although iminium ion $\mathbf{1 2}$ did not undergo the Mannich cyclization, the corresponding iminium ion $\mathbf{2 8}$ (from activation of lactam 27) proved to be much more reactive, thus confirming that triflyl iminium ions (28) are more electrophilic than iminium ions of lower oxidation state (12) in our reaction cascade.

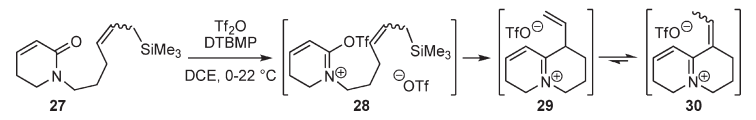

(19) We observed the following decreasing order of nucleophilicity: allylstannane $>$ allylsilane with non-substituted nucleophilic carbon (cf. 1a) > allylsilane with substituted nucleophilic carbon (cf. 9) > vinylsilane. This order is in accord with Mayr's calculated nucleophilicity of carbon nucleophiles. See: Mayr, H.; Kempf, B.; Ofial, A. R. Acc. Chem. Res. 2003, 36, 66.

(20) We do not have a clear explanation to why, in this case, we observed a competitive deprotonation to 16 . We could presume that the vinyl group induces a conformation in which the indicated proton in $\mathbf{2 6}$ (Scheme 3) is acidified due to a better alignment with the p orbital of the carbocation. For another recent example of competition between desilylation and deprotonation, see: Lee, M. H.; Nieto-Oberhuber, C.; Shair, D. M. J. Am. Chem. Soc. 2008, 130, 16864.

(21) Among the two branches, the hypothetical initial cyclization of the allylsilane branch would have generated an iminium intermediate almost identical to the ones produced for the cyclization of 1a and $\mathbf{1 1 .}$ Because no TMS group was observed in the products for the cyclization of 1a and 11 and because the allylstannane is calculated to be more nucleophilic than the allylsilane (ref 19), we could presume that the reaction path for the cyclization of $\mathbf{1 4}$ is as described in Scheme 3. 
onto the face of the iminium ion opposed to the vinyl group $\left(\mathrm{C}_{3}\right)$ in 25 (Scheme 3$)^{23}$

Scheme 3. Deprotonation versus Desilylation Observed for Formamide 14

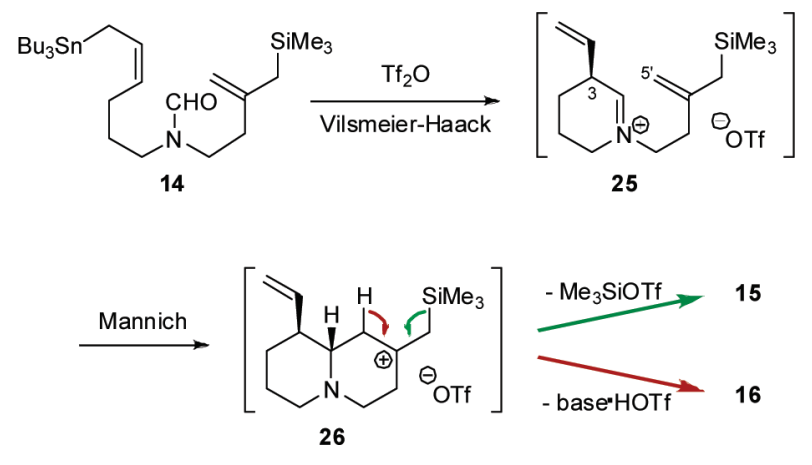

The successful cyclization of $\mathbf{1 4}$ to $\mathbf{1 5}$ and $\mathbf{1 6}$ indicated that the second cyclization could tolerate steric hindrance generated by substitution at $\mathrm{C}_{3}$ on the iminium ion intermediate (cf. 25, Scheme 3), as long as the remaining branch is not substituted at the nucleophilic carbon $\mathrm{C}_{5^{\prime}}$. This is also true when an indole is used as the nucleophile (substrate 17, entry 8, Table 1), considering that the indole undergoes the initial cyclization due to its higher nucleophilicity compared to allylsilanes. ${ }^{19}$ Product $\mathbf{1 8}$ has the framework of natural product ajmaline (Figure 1).

Activation of substrate $\mathbf{1 9}$ afforded the iminium intermediate 20 after conjugation (entry 9, Table 1). ${ }^{24} \mathrm{We}$ hoped that the latter would undergo a [3.3] rearrangement and a subsequent trapping to afford the expected quinolizidine. ${ }^{25}$ Unfortunately, no further reaction with iminium ion $\mathbf{2 0}$ was observed after the first cyclization.

Finally, in order to increase the level of control on the order of nucleophilic cyclizations, we tested the possibility of using a latent nucleophile. Hence, less reactive substituted allylsilanes such as in $\mathbf{9}$ could be used in the first Vilsmeier-Haack-type cyclization, and the second cyclization (Mannich) might still be performed upon

(22) When the reaction was run at $-45^{\circ} \mathrm{C}$, the $15: 16$ ratio increased to 1:9, but the global yield was lower ( $31 \%)$; in the absence of DTBMP, the global yield decreased to $48 \%$ and the $15: 16$ ratio was lowered to $1: 0.5$.

(23) Signals at positions $C_{2}$ and $C_{3}$ in $\mathbf{1 5}$ were identified from the HMQC spectrum, and relative stereochemistry was determined from coupling constants in the ${ }^{1} \mathrm{H}$ NMR spectrum. See the Supporting Information.

(24) The stereochemistry of the trisubstituted double bond in $\mathbf{2 0}$ was determined to be cis, as established by NOESY experiments conducted on piperidine $\mathbf{3 1}$ obtained after reduction of the iminium ion.



(25) Attempted rearrangement with iminium 20:



unmasking of the latent nucleophile. This way, we could circumvent reactivity issues such as those encountered with iminium intermediates $\mathbf{1 0}, \mathbf{1 2}$, and $\mathbf{2 0}$ that bear a second nucleophile that is not reactive enough to undergo the Mannich cyclization (entries 4, 5, and 9, respectively). To test this idea, we used an alkyne as a masked nucleophile ${ }^{26}$ that cyclized upon addition of a halide source such as tetrabutylammonium iodide (entry 10) or tetrabutylphosphonium bromide (entry 11). In the latter entry, we deliberately chose a TBS enol ether for the Vilsmeier-Haack cyclization: this type of nucleophile is generally not well suited for tandem nucleophilic cyclizations onto activated amides because, after initial addition, the resulting iminium ion intermediate is deactivated due to electrodonation of the silyloxy group (cf. 5, Scheme 2). Interestingly, in the present case, the bicyclo[2.2.2]octane unit that is formed upon the VilsmeierHaack cyclization prevents conjugation of the silyl enol ether with the iminium ion. The latter was then successfully trapped, after spontaneous cleavage of the TBS group, ${ }^{15}$ with the pendent alkyne upon addition of a bromide salt. ${ }^{11}$ The topology of the bicycle allowed for the use of otherwise incompatible nucleophile combinations for the tandem cyclizations. The use of an alkyne as latent nucleophile furnished quinolizidines $\mathbf{2 2}{ }^{27}$ and $\mathbf{2 4}$ bearing vinylic halides that could potentially be used for further functionalization.

In conclusion, we successfully developed a novel cascade of cyclizations for the rapid and convergent synthesis of substituted quinolizidines. Tertiary and quaternary centers at the ring junction were efficiently prepared. The reaction tolerates a broad range of tethered carbon nucleophiles. Alkynes used as latent nucleophiles allowed for a perfect control of the sequence of cyclizations and generated vinylic halides, which are useful coupling partners. Further applications of this tandem reaction to the syntheses of complex alkaloids are currently under investigation.

Acknowledgment. This research was supported by the Natural Science and Engineering Research Council (NSERC) of Canada, the Fonds Québécois pour la Recherche sur la Nature et les Technologies (FQRNT), the Canadian Fund for Innovation, and the Université de Sherbrooke. Boehringer Ingelheim, NSERC, and FQRNT doctoral fellowships to R.L.-G. are also gratefully acknowledged.

Supporting Information Available. Experimental procedures including detailed synthesis of cyclization precursors, spectral data, and characterization for all relevant compounds. This material is available free of charge via the Internet at http://pubs.acs.org.

(26) (a) Overman, L. E.; Sharp, M. J. J. Am. Chem. Soc. 1988, 110, 612. (b) Overman, L. E.; Rodriguez-Campos, I. M. Synlett 1992, 995.

(27) The low yield in $\mathbf{2 2}$ (entry 10) could be due to the lower electrophilicity of the conjugated iminium intermediate (compared to the iminium intermediate in the formation of $\mathbf{2 4}$, entry 11). Other sources of halogen were tested, but yields were not improved. 